\title{
Deep Transfer Learning Approach for Detection of Covid-19 from Chest X-Ray Images
}

\author{
Lakshay Goyal \\ School of Computer Science \\ UPES, Dehradun, India
}

\author{
Nitin Arora \\ Electronics \& Computer Discipline \\ IIT, Roorkee, India
}

\begin{abstract}
COVID-19 is an irresistible illness brought about by the most as of late found coronavirus. This new infection and malady were obscure before the episode started in Wuhan, China, in December 2019 [1]. COVID-19 is currently a pandemic influencing numerous nations universally.Analyzing after which diagnosing is presently a significant assignment. The Coronavirus (COVID-19) pandemic has led to the most significant number of employees globally bound to work remotely. With the advancements in computer algorithms and particularly Artificial Intelligence, the detection of this type of virus in the early stages will help in the speedy recovery and assist in freeing the pressure off healthcare operations. This paper focuses on the arrangement which can help in the examination of COVID-19 with a regular chest X-rays utilizing deep learning techniques. The primary approach is to collect all the possible images for COVID-19 that exist and use the Convolutional Neural Network to generate more images to help in the detection of the virus from the usable $\mathrm{X}$ rays images with the highest accuracy possible. The number of images in the collected dataset is 748 images for three different types of classes. The classes are the COVID-19, normal, pneumonia bacterial. Three deep transfer models are selected in this research for investigation. The models are the VGG19, VGG16, and Restnet50.
\end{abstract}

\section{Keywords}

COVID-19, Deep Learning, Transfer Learning, Convolutional Neural Network

\section{INTRODUCTION}

Coronavirus disease 2019 (COVID-19) is an infectious disease caused by severe acute respiratory syndrome coronavirus 2 (SARS-CoV-2). It was first recognized in December 2019 in Wuhan, China, and has since spread all around, bringing about a continuous pandemic. Starting on 24 May 2020, more than 5.34 million cases have been accounted for across 188 nations and domains, bringing about more than 342,000 passing. More than 2.13 million individuals have recovered. Common symptoms include fever, cough, fatigue, shortness of breath, and loss of smell and taste. While most of the cases bring about gentle manifestations, some advancement to intense respiratory trouble disorder (ARDS) likely hastened by a cytokine storm, multi-organ

disappointment, septic stun, and blood clumps. The time from presentation to the beginning of side effects is ordinarily around five days yet may run from two to fourteen days. The virus is primarily spread between people during close contact, most often via small droplets produced by coughing, sneezing, and talking.The beads, for the most part, tumble to the ground or onto surfaces as opposed to going through the air over significant distances. Less ordinarily, individuals may get tainted by contacting a defiled surface and afterward contacting their faces. It is generally infectious during the initial three days after the beginning of indications. However, the spread is conceivable before manifestations show up, and from individuals who don't show side effects. The standard strategy for determination is by ongoing converse translation polymerase chain response (RRT-PCR) from a nasopharyngeal swab. Chest CT imaging may likewise be useful for confidence in people where there is a high doubt of contamination dependent on manifestations and hazard factors; be that as it may, rules don't suggest utilizing CT imaging for routine screening.

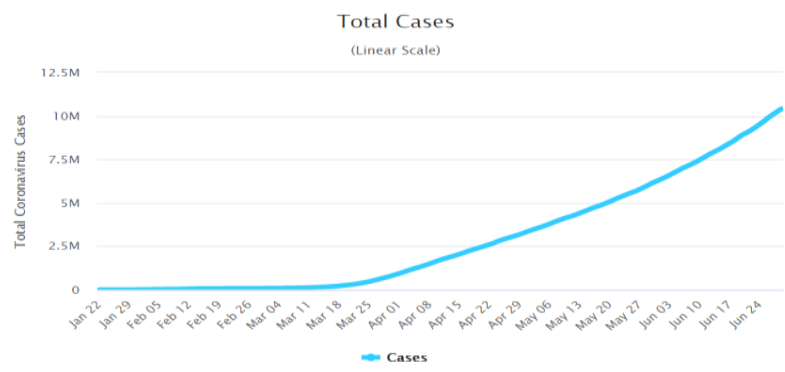

Fig 1: Total cases of COVID-19 worldwide

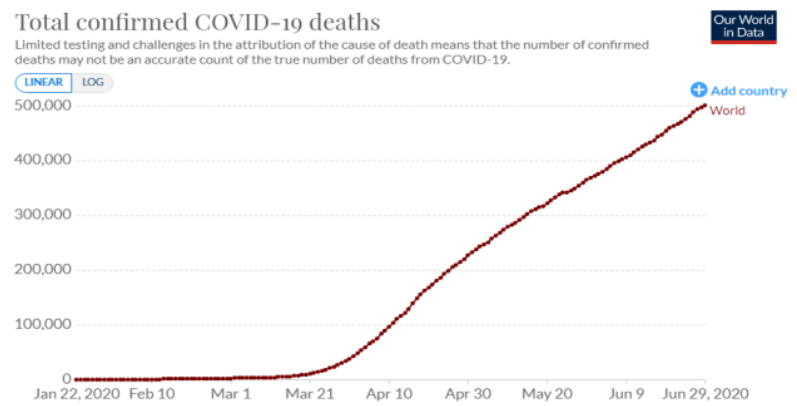

Fig 2: Total Deaths due to COVID-19 worldwide

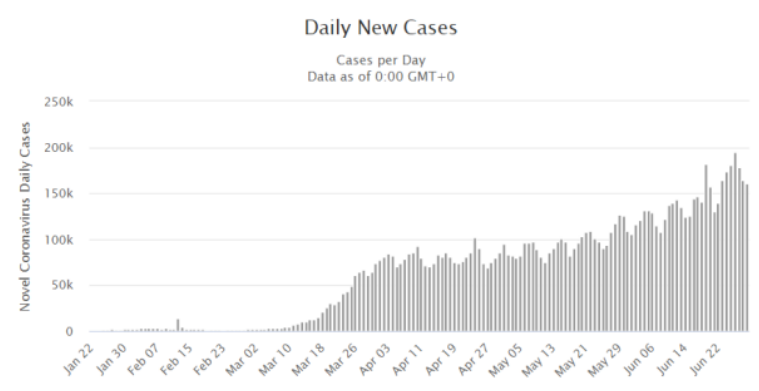

Fig 3: Daily New Cases 


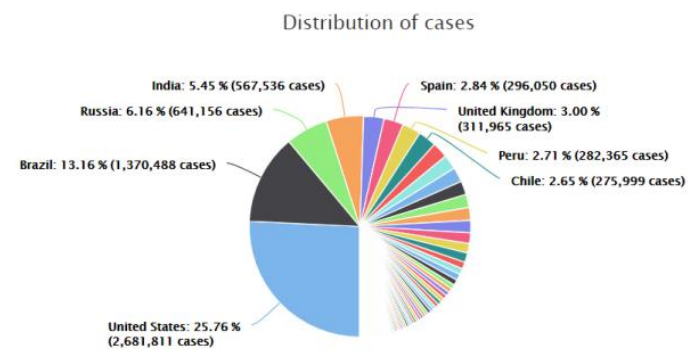

Fig 4: Distribution of Cases Worldwide

As indicated by the World Wellbeing Association, there are no available immunizations nor explicit antiviral medications for COVID-19. On 1 May 2020, the US gave crisis use approval to the antiviral redeliver for individuals hospitalized with extreme COVID- 19. The board includes the treatment of manifestations, strong consideration, detachment, and trial measures. The WorldHealth Organization (WHO) [2] pronounced the COVID- 19 episode a general wellbeing crisis of universal concern (PHEIC) on 30 January 2020 and a pandemic on 11 Walk 2020. Nearby transmission of the illness has happened in many nations over every one of the six WHO districts.

\section{RELATED WORK}

This part leads a review of the ongoing logical looks into for applying AI and deep learning in the field of clinical pneumonia and coronavirus X-rays arrangement. Classical style picture arrangement stages can be partitioned into three principal stages: image preprocessing, feature extraction, what's more, include order. Stephen et al. [6] proposed another investigation of grouping. They identified the nearness of pneumonia from an assortment of chest X-rays picture tests dependent on a ConvNet model prepared without any preparation dependent on the dataset. The results got were training loss $=12.88 \%$, training accuracy $=95.31 \%$, validation loss $=18.35 \%$, and validation accuracy $=93.73 \%$. In, the [7] Creators presented an initial finding framework from Pneumonia chest X-rays pictures in light of Xception and VGG16. In this examination, a database containing roughly 5800 frontal chest X-rays pictures presented by Kermany et al. 1600 typical case, 4200 up-ordinary pneumonia case in the Kermany X-rays database. The preliminary results indicated that the VGG-16 system better than Xception coordinates with a classification rate of $87 \%$. Since Xception arranges better than the VGG-16 system by sensitivity $85 \%$, precision $86 \%$ and recall $94 \%$. Xception arrangesit is increasingly well suited for characterizing X-rays pictures than the VGG-16 system. Varshniet al.[8]proposed pre-prepared ConvNet models (VGG-16, Xception, Res50, Thick 121, and Thick 169) as highlight extractors followed by various classifiers(SVM, Arbitrary Woodland, k-closest neighbors, Credulous Bayes) for the identification of ordinary and unusual pneumonia Xrays pictures. The prosiest utilized ChestX-ray 14 presented by Wang et al. Chouhanet al.[9] presented a profound outfit model that consolidates yields from all profound exchange models for the grouping of pneumonia utilizing the implication of profound learning. The Guangzhou Clinical Center database presented an aggregate of roughly $5200 \mathrm{X}-$ rayspictures, isolated to $1300 \mathrm{X}$-rays ordinary, $3900 \mathrm{X}$-rays irregular. The proposed model arrived at a misscharacterization error of $3.6 \%$, with an affectability of $99.6 \%$ on test information from the database. Ref. [10]proposed a Compacted Detecting (CS) with a profound exchange learning model for the programmed arrangement of pneumonia on $\mathrm{X}$ - rays pictures to help the clinical doctors. The dataset utilized for this work contained roughly $5850 \mathrm{X}$-rays information of two classes (anomalous/typical) acquired from Kaggle. Complete recreation results have indicated that the proposed approach identifies the arrangement of pneumonia (strange/ordinary) with $2.66 \%$ miss-classification. In this examination, it presented an exchange of profound learning models to arrange COVID-19 X-rays pictures. To include embracing X-rays pictures of the chest to the convolutional neural system, inserted the clinical $\mathrm{X}$-rays pictures utilizing GAN to produce X-rays pictures. From that point onward, a classifier is utilized to troupe the yields of the arrangement results. The proposed move model was assessed on the proposed dataset.

\section{METHODOLOGY}

Transfer learning is a machine learning approach where a model developed for a task is reused as the starting point for a model on a second task.We transfer the weights that a network has learned at one job to a new task."Transfer learning has several benefits, but the main advantages are saving training time, better performance of neural, and not needing a lot of data.In computer vision, transfer learning is usually expressed via the use of pre-trainedmodels. Mostly pre-trained models used in transfer learning are based on large convolutionalneuralnetworks (CNN).In this paper, the network architecture used to perform classification with transfer learning is VGG19, and ImageNet weights have been used, which is when compared to VGG16, ResNet50.For Detection, we are comparing the three different $(\mathrm{CNN})$ architecture:

\subsection{VGG19}

VGG19 is pre-trained on a large dataset (ImageNet) to build image representations. The model reaches around $92.7 \%$ top-5 test accuracy in ImageNet. It achieves competitive classification accuracy compared to more complicated nets, even though at the expense of more slow evaluation speed and a lot bigger net size.It is significant for its amazingly basic structure, with all the convolutional layers having a kernel size of $3 \times 3$ with stride 1 . There are five sets of conv layers, 2 of them have 64 filters, the next set has twoconv layers with 128 filters, the next set has fourconv layers with 256 filters, and the next two sets have fourconv layers each, with 512 filters. There are max-pooling layers in each set of conv layers. Max-pooling layers have $2 \times 2$ filters with a stride of 2 (pixels). The output of the last pooling layer is flattened and is fed to a fully connected layerthat is utilized for classification with 4096 neurons. The output goes to another fully connected layer with 4096 neurons, whose output is fed into another fully connected layer with 1000 neurons. All these layers are ReLU activated. Atlast, there is a softmax layer.There are around138,357,544parameters in whichTrainable parametersare 138,357,544 andNon-trainable parametersare 0 .
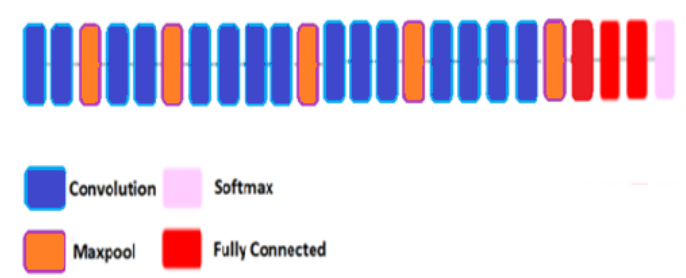

Fig 5: VGG19 Architecture 


\subsection{VGG16}

It is a convolutional neural network architecture. It has 16 layers. Its layers consist of Convolutional layers, Max Pooling layers, Activation layers, Fully connected layers. There are 13 convolutional layers, 5 Max Pooling layers, and 3 Dense layers, which sum up to 21 layers but only 16 weight layers.VGG-16 network is trained on the ImageNet dataset, which has above 14 million images and 1000 classes and gains $92.7 \%$ top-5 accuracy. Three fully connected layers follow the convolutional layers. The width of the network starts at a small value of 64 and increases by a factor of 2 after every pooling layer. However, VGG16 consists of 140 million parameters.
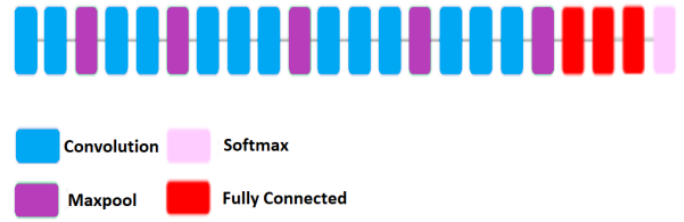

Fig6: VGG16 Architecture

\subsection{ResNet50}

ResNet, short for Residual Networks, is a standard neural network. It is a convolutional neural network that is 50 layers deep. The focused thought of ResNet is presenting a supposed "character alternate way association" that avoids at least one layer. There are around 23,587,712 parameters in which Trainable parameters are 23,534,592 and Non-trainable parameters are 53,120 .

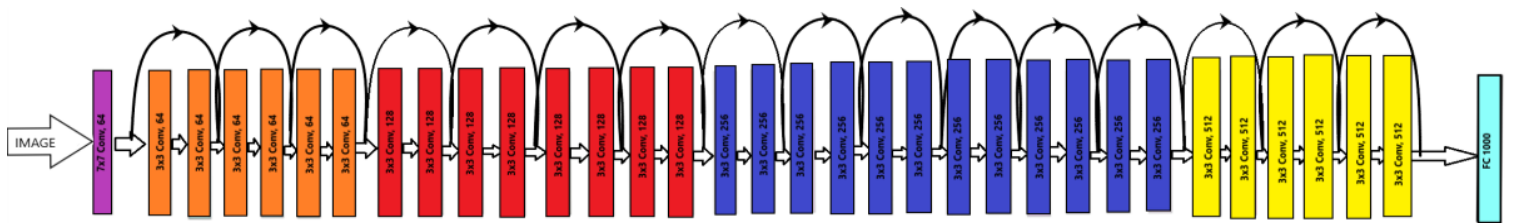

Fig7:ResNet50 Architecture

\section{EXPERIMENTAL RESULTS}

\subsection{Experimental Setup}

Experiments were carried out on a machine having a Ryzen 7 Quad-Core, 16 GB RAM, 6 GB RAM of NVIDIA Geforce GTX 1660 Ti Max-Q, and Windows 10 OS. All the programs were written in Python, where the software stack consists of scikit-learn and Keras with the TensorFlow backend.The networks were trained up to 50 epochs, and for the optimization, Adam optimizer is used.

\subsection{Datasets}

The dataset is organized into two folders (train, test) and contains sub-folders for each image category (COVID19/normal/pneumonia bacterial). There are $748 \mathrm{X}$-ray images and three types (COVID-19/normal/pneumonia bacteria). The

number of images for each class is presented in Table 1. All images that come under the category of multispectral images means those images whose features lie beyond the visible spectrum. The images were downloaded from Kaggle. Thus the proposed approach fits very well in this dataset as the features will be more than the usual.

Table 1.Number of images for each class in the Dataset

\begin{tabular}{|c|c|c|c|c|}
\hline $\begin{array}{c}\text { Dataset/CI } \\
\text { ass }\end{array}$ & $\begin{array}{c}\text { COVI } \\
\text { D-19 }\end{array}$ & $\begin{array}{c}\text { NORM } \\
\text { AL }\end{array}$ & $\begin{array}{c}\text { PNUEMON } \\
\text { IA }\end{array}$ & $\begin{array}{c}\text { TOTA } \\
\text { L }\end{array}$ \\
\hline Train & 200 & 240 & 150 & 590 \\
\hline Test & 50 & 60 & 48 & 158 \\
\hline Total & 250 & 300 & 198 & 748 \\
\hline
\end{tabular}

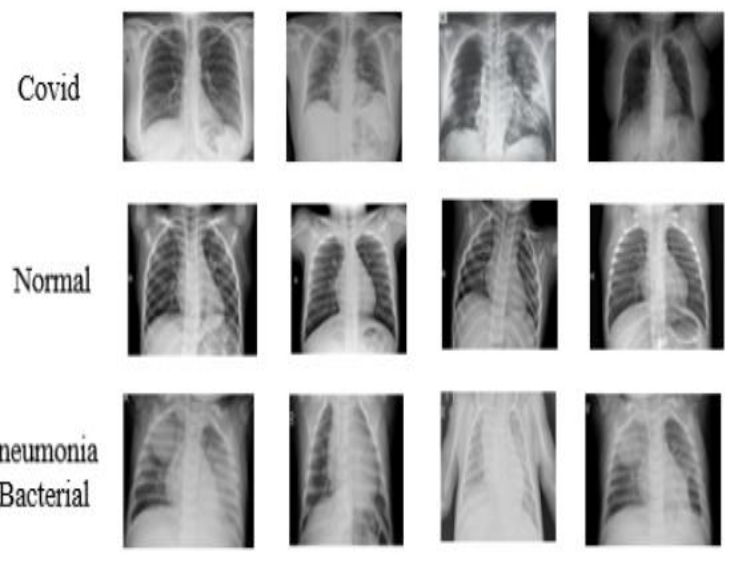

Fig 8: Sample of X-Ray Images used in this Research

For the training and testing part, the dataset is divided into $75 \%$ and $25 \%$, which means $75 \%$ of the data is used for training, and $25 \%$ is used for testing.

Table 2: Accuracy Measures of VGG19

\begin{tabular}{|c|c|c|c|}
\hline & PRECISION & RECALL & $\begin{array}{c}\text { F1- } \\
\text { SCORE }\end{array}$ \\
\hline COVID-19 & 0.99 & 0.99 & 0.99 \\
\hline PNEUMONIA & 0.98 & 0.99 & 0.98 \\
\hline NORMAL & 1.0 & 0.99 & 0.99 \\
\hline
\end{tabular}

As shown, the F1 Score, which is the weighted average of Precision and Recall, is a measure of test accuracy, which can be calculated by Eq. (1)

F1 Score $=\frac{2 \times \text { Precision } \times \text { Recall }}{\text { Precision }+ \text { Recall }}$ 


\subsection{Comparison of the VGG19 with VGG16 and ResNet50}

LOSS AND ACCURACY ON VGG19

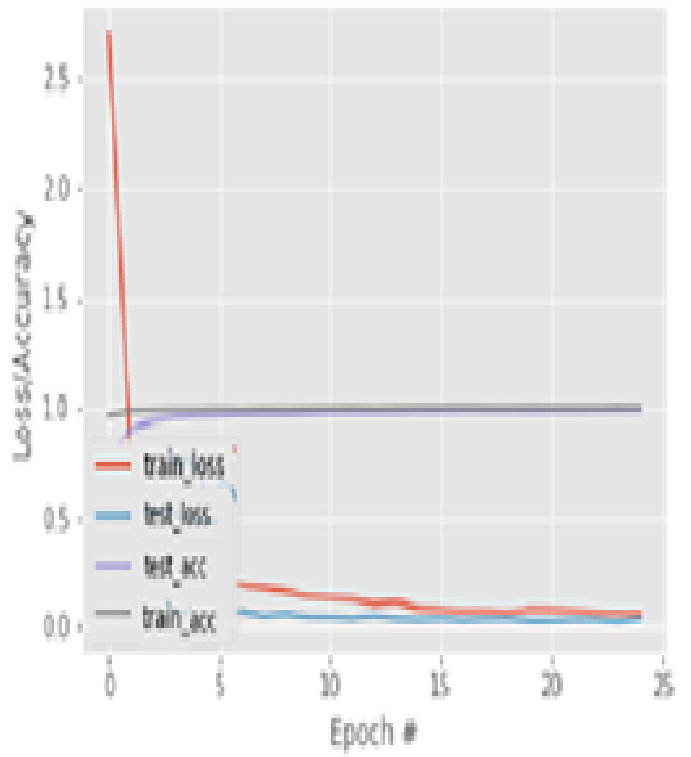

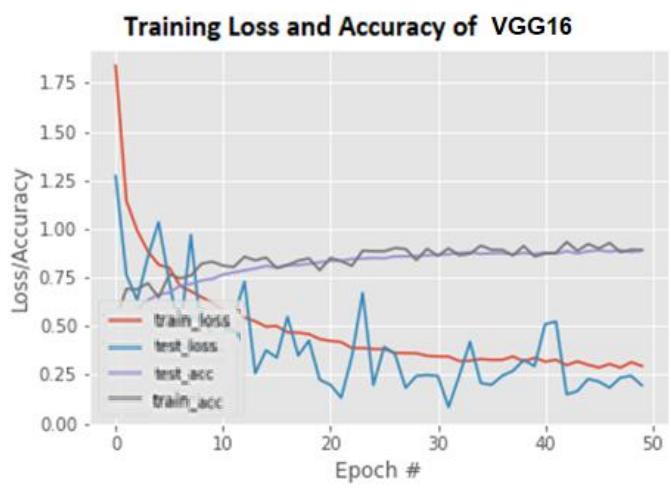

LOSS AND ACCURACY ON ResNet50

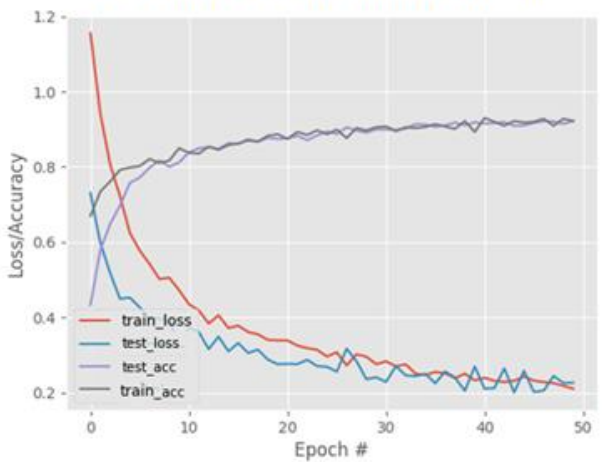

Fig 9: Loss and Accuracy of Comparison of VGG19 with VGG 16 and ResNet 50

Table 3: Comparison of Different CNN Models

\begin{tabular}{|c|c|c|c|c|c|}
\hline MODEL & SIZE(M) & LAYERS & MODEL DESCRIPTION & $\begin{array}{c}\text { TRAINING } \\
\text { ACCURACY }\end{array}$ & $\begin{array}{c}\text { TEST } \\
\text { ACURACY }\end{array}$ \\
\hline VGG19 & 560 & 19 & 16 conv +3 fc layers & $98.79 \%$ & $98.12 \%$ \\
\hline VGG16 & 528 & 16 & 13 conv +3 fc layers & $95.98 \%$ & $93.67 \%$ \\
\hline RESNET-50 & 100 & 50 & 49 conv +1 fc layer & $92.35 \%$ & $90.15 \%$ \\
\hline
\end{tabular}

\section{CONCLUSION}

As VGG19 has better performance when compared with other CNN when used ImageNet weights. The dataset consists of three classes, and, thus, the transfer learning strategy has been used. The types are the COVID, normal, pneumonia bacterial. In the first scenario, the VGG16 was selected to be the central deep transfer model, as it obtained $93.67 \%$ in testing accuracy. In the second synopsis, the Resnet50 was chosen to be the deep transfer model as it achieved $90.15 \%$ in testing accuracy. In contrast, in the final scenario, VGG19 was selected to be the primary deep transfer model as it made Training Accuracy: 98.79\% and 98.12\% Testing accuracy.

\section{REFERENCES}

[1] https://www.hindustantimes.com/world-news/covid-19virus-accidently-leaked-by-intern-at-wuhan-lab-says-usmedia/story-15OJrBp6t9zdY9baRY8P1N.html

[2] https://www.who.int/healthtopics/coronavirus\#tab=tab_1

[3] https://www.worldometers.info/coronavirus/coronaviruscases/
[4] https://www.worldometers.info/coronavirus/coronavirusdeath-toll/

[5] https://www.worldometers.info/coronavirus/

[6] Stephen, O.; Sain, M.; Maduh, U.J.; Jeong, D.-U. An Efficient Deep Learning Approach to Pneumonia Classification in Healthcare. J. Healthc. Eng. 2019, 2019 , 4180949.

[7] Ayan, E.; Ünver, H.M. Diagnosis of Pneumonia from Chest X-ray Images Using Deep Learning. In Proceedings of the 2019 Scientific Meeting on ElectricalElectronics \& Biomedical Engineering and Computer Science (EBBT), Istanbul, Turkey, 24-26 April 2019; pp. 1-5.

[8] Varshni, D.; Thakral, K.; Agarwal, L.; Nijhawan, R.; Mittal, A. Pneumonia Detection Using CNN based Feature Extraction. In Proceedings of the 2019 IEEE International Conference on Electrical, Computer and Communication Technologies (ICECCT), Coimbatore, India, 20-22 February 2019; pp. 1-7. 
[9] Chouhan, V.; Singh, S.K.; Khamparia, A.; Gupta, D.; Tiwari, P.; Moreira, C.; Damaševi cius, R.; de Albuquerque, V.H.C. A Novel Transfer Learning Based Approach for Pneumonia Detection in Chest X-ray Images. Appl. Sci. 2020, 10, 559 Singhal, T. A Review of Coronavirus Disease-2019 (COVID-19). Indian J. Pediatrics 2020, 87, 281-286.

[10] Islam, S.R.; Maity, S.P.; Ray, A.K.; Mandal, M Automatic Detection of Pneumonia on Compressed Sensing Images using Deep Learning. In Proceedings of the 2019 IEEE Canadian Conference of Electrical and Computer Engineering (CCECE), Edmonton, AB, Canada, 5-8 May 2019; pp. 1-4. I. D. Apostolopoulos and T. Bessiana, "Covid-19: Automatic detection from X-Ray images utilizing Transfer Learning with Convolutional Neural Networks," Phys. Eng. Sci. Med., Mar. 2020.

[11] P. Kumar and S. Kumari, "Detection of coronavirus Disease (COVID-19) based on Deep Features," preprints.org, no. March, p. 9, Mar. 2020. 